\title{
Ein Tag Notfalldienst im Sommer 2001
}

M. Gassner

Arzt sein wird heute immer schwieriger. Wir haben unseren Beruf gewählt, um jedem kranken Menschen $\mathrm{zu}$ helfen, wie unsere Kollegen seit Jahrtausenden und in allen Kulturen.

Wo sucht ein Mensch Hilfe, der heute plötzlich krank wird? Welche Hilfe können wir Ärzte ihm bieten. Welche Rolle spielt dabei der Arzt im Notfall? Wie können wir die Realität der ärztlichen Versorgung heute messen? Sind z.B. meine Notfall-TriageEntscheide zu riskant oder zu teuer? Welche technischen Hilfsmittel sind dafür unbedingt notwendig?

\section{Geographische Lage und Infrastruktur}

Wie seit Jahrzehnten versorgen 2 Ärzte über das Wochenende und am Donnerstag den Notfalldienst im Bezirk Werdenberg (206 km², 33000 Einwohner). Gelegentlich werden wir auch von Patienten aus dem Fürstentum Liechtenstein und anderen Regionen konsultiert.

Das Regionalspital ist 600 Meter von der Praxis entfernt.

Ein Institut für Laboruntersuchungen steht in einer Entfernung von etwa $8 \mathrm{~km}$ zur Verfügung, wo auch notfalls entsprechende Analysen durchgeführt werden könnten.

Die nächste Apotheke ist $4 \mathrm{~km}$ entfernt. Rezepte werden generell selten ausgestellt. Sämtliche Medikamente werden in der Praxis abgegeben.

Für Vergleiche mit anderen Ärzten, aber auch mit anderen Regionen, sind Angaben zur vorhandenen Infrastruktur der individuellen Praxis notwendig: Im Notfalldienst steht keine weitere Hilfsperson zur Verfügung. Sämtliche Telefonanrufe werden vom Notfallarzt selbst entgegengenommen, mit Ausnahme der Zeit während eines Hausbesuchs oder während einer Wundversorgung. In dieser Zeit übernimmt seit über zwanzig Jahren meine Frau die telefonische Triage.

Apparate (in diesem Notfalldienst benützt): Röntgenanlage (3x), Mikroskop, (1x); unbenutzt: EKG, Reflotron, Spirometer. Ein Defibrillator steht nicht zur Verfügung.

Korrespondenz:

Dr. med. Markus Gassner

Spitalstrasse 8

CH-9472 Grabs

\section{Kasuistik}

Die chronologische Aufzeichnung aller Notfallkonsultationen an einem Samstag im Sommer 2001 in meiner Praxis ist ein schlichter Versuch, die Situation heute aufzuzeigen.

1938 w: akuter Schulterschmerz seit der letzten Nacht. Am Vortag Sturz auf der Strasse.

Objektiv exquisite Druckdolenz im Bereiche der Supraspinatussehne, Schürzengriff problemlos, Nackengriff mühsam mit Schmerzen. Röntgen: Keine Fraktur.

Diagnose: Impeagement-Verletzung re Schulter.

Therapie: Mitella, Ponstan.

Verlauf: nach 2 Wochen noch mässig eingeschränkte Schulterbeweglichkeit.

1948 w: Husten seit einer Woche mit Schnupfen trotz Similasan.

Objektiv trockener Reizhusten, Lunge unauffällig.

Diagnose: viraler Infekt mit akuter Bronchitis.

Therapie: Dafalgan, Codipront.

Verlauf: nach 2 Tagen Besserung, wünscht noch Practoclyss zur Darmreinigung.

1915 w: Sohn bringt Kurzbericht vom Spital, Analgesie mit Dafalgan $3 \times 500 \mathrm{mg}$ ungenügend.

Diagnose: Schambeinastfraktur links vor 4 Tagen.

Therapie: Optifen, Tramaltropfen.

Verlauf: konnte so 10 Tage zu Hause gepflegt werden, musste dann doch noch aus pflegerischen Gründen hospitalisiert werden.

1930 w: Gemeindeschwester wünscht Hausbesuch (Distanz $13 \mathrm{~km}$ ) wegen akutem Schmerz im Bereiche der rechten Hüfte bei Diabetes.

Objektiv klinisch Zeichen einer:

Diagnose: Schenkelhalsfraktur.

Therapie: notfallmässige Hospitalisation.

Verlauf: gleichentags Gamma-Nagel-Osteosynthese, komplikationsloser Verlauf.

1989 m: Mutter besorgt wegen Hautausschlag seit den Badeferien am Meer.

Objektiv generalisiertes makulopapullöses, nicht juckendes Exanthem ohne Pruritus und ohne weitere subjektive Beschwerden.

Diagnose: virales Exanthem.

Therapie: keine.

1916 w: kommt wegen "Knacks am rechten Puls», plötzlich nach der Dusche beim Abtrocknen des Rückens.

Objektiv Druckdolenz im Bereiche des MP-I-Gelenks rechts. Röntgen: massive arthrotische Veränderungen im Bereiche des Handgelenkes, insbesondere des MP-I-Gelenks. 
Diagnose: traumatisierte Rhizarthrose.

Therapie: elastische Binde, Dafalgan.

Verlauf: am andern Tag: noch Schmerzen, aber zufrieden, will nichts weiteres.

1994 m: Zehe in Küchentüre eingeklemmt.

Objektiv: Blutung am Nagelfalz. Röntgen: Keine Fraktur, keine AP für Epiphysenlösung.

Diagnose: Quetschung Zehe.

Therapie: Ponstan 250, Verband.

Verlauf: keine Komplikation.

1990 w: Durchfall eitrig-blutig, Temperatur 37,8, AZ gut, Abgang eines Koagulums, Mens bisher keine, Bauchkrämpfe. Die Eltern sind hier in den Ferien wollen heute heimkehren.

Empfehlung: sollen ihre Tochter diesbezüglich in ihrer eigenen Region abklären zu lassen, entweder beim Hausarzt oder beim dortigen Notfallarzt, keine Konsultation.

1996 m: Ein Vater aus dem Balkan (gemäss seinem Akzent) will wissen, ob er seinem 5jährigen Sohn mit Angina und Antibiotikum vom Hausarzt noch wie viel Dafalgan zur Fiebersenkung geben darf. Zäpfchen à $300 \mathrm{mg}$.

Antwort: alle 6 Stunden eines. Kontrolle bei Auffälligkeit des Kindes, Name nicht genannt.

\section{Diskussion}

Der ärztliche Notfalldienst hat in erster Linie die Aufgabe einer notfallmässigen Triage: Sofortige Weiterweisung, Abwarten, probatorische Behandlung. Generelle Richtlinien für die Kunst der Triage in unterschiedlichen zeitlichen und regionalen Gegebenheiten sind kaum möglich.

Grosse differenzialdiagnostische Überlegungen oder Abklärungen sind nicht sinnvoll, weil hierzu der Hausarzt zuständig ist, welcher in zwei Tagen den Patienten wieder übernimmt und ihn kennt.

Die technologische Entwicklung im Medizinalbereich führte im Verlauf der letzten Jahrzehnte zu einer zunehmenden Diskrepanz (Individualisierung der ärztlichen Praxis infolge Spezialisierung) aber auch $\mathrm{zu}$ einer Zentralisierung diagnostischer und therapeutischer Möglichkeiten (z.B. Autounfälle, Herzinfarkt, Apoplexie). Die Intensivpflegestationen der Spitäler wurden mobil und über Telekommunikation vernetzt (Kardiomobil, Notarzt, Ambulanz, REGA, Sanitätsnotruf). Unklar bleibt, welche apparative Infrastruktur des Notfallarztes auf Kosten von wem sinnvoll angeschafft und unterhalten werden soll.

Welcher Arzt kann, muss sich einen Defibrillator anschaffen, funktioniert er dann auch, wenn er nur alle zwanzig Jahre einmal gebraucht wird? Gilt dies auch für den Notfallarzt, welcher weiter von einem Spital entfernt ist?
In den letzten zwanzig Jahren hatte ich lediglich einmal vor zehn Jahren die Gelegenheit, eine Reanimation durchzuführen: In der benachbarten Metzgerei erlitt ein Mann an einem Samstagvormittag ein Kammerflimmern (nicht im Notfalldienst). Da damals auch das Spital über keinen Defibrillator in der Ambulanz verfügte, musste die Herzmassage in der Ambulanz bis ins Spital fortgesetzt werden. Diese war erfolgreich. Ich sah den Patienten nie in der Praxis, jedoch vor zwei Jahren zufällig. Er überlege sich, etwas frühzeitig in Pension zu gehen.

Eine Reanimation ist selten erfolgreich, insbesondere nachts. Ich bin zwar gleichzeitig mit der Ambulanz alarmiert ein paar Minuten schneller beim Patienten, was aber wenig zum Überleben beiträgt.

Auswärtige Laboruntersuchungen werden im Notfalldienst selten veranlasst. Bei sehr dringlichen lebensbedrohlichen Entscheidungen (z. B. Herzinfarkt, Thrombose, Embolie) nützt dies wenig. Es muss "gefühlsmässig" rasch über die Notwendigkeit einer Hospitalisation entschieden werden.

Irrelevant im Notfalldienst scheint mir das Internet, bei welchem Fall der Kasuistik hätte mir dies geholfen?

Medikamente: Es macht speziell im Notfalldienst wenig Sinn, ein Rezept auszustellen. Die Abgabe des Medikamentes in der Praxis ist effizienter. Der Arzt kann die Applikation selbst kontrollieren, was bei Medikamenten zur Behandlung von Atemwegserkrankungen (z.B. Nasenspray, Dosieraerosol) wichtig ist. Gleichzeitig kann auch die Wirkung z.B. bei Asthma unmittelbar beurteilt werden. Hospitalisationen können so vermieden werden.

Auch die Bedürfnisse des Patienten haben sich geändert. Immer mehr Patienten melden sich am Telephon mit der Frage an: «Sie haben Notfalldienst - oder?»

Die Konsultation im Notfalldienst ist kein medizinisches Konsilium. Der Notfalldienst wird zunehmend offen oder unterschwellig von Patienten für eine "zweite Meinung" missbraucht.

Neu und ausserordentlich belastend für einen betroffenen Arzt sind juristische Probleme im Zusammenhang mit der Haftpflicht. Wir haben hier bereits amerikanische Verhältnisse. Ein Schicksalsschlag wird heute als solcher nicht mehr akzeptiert. Es wird immer mehr versucht, auf Kosten der Haftpflichtversicherung des Arztes wenigstens noch einen finanziellen Vorteil zu ergattern. Dies belastet die Arbeit des Arztes im Notfalldienst. Triageentscheidungen werden so zunehmend delegiert (vermehrte unnötige Hospitalisationen). Damit werden direkte und schwer schätzbare indirekte Kosten verursacht.

Für Haftpflichtversicherungen sind zudem bei entsprechenden prozessualen Abklärungen die Zeitaufwendungen eines betroffenen Arztes mit den massiven Frustrationen für die ganze Familie völlig irrelevant. Dies ist ein wichtiger Grund für die zunehmende Verschlechterung der «Befindlichkeit von Grundversorgern". 